Produto \& Produção, vol. 11, n. 2, p. 55 - 66, jun. 2010

$\mathbf{P P}$

\title{
Estudo de Fatores que Influenciam o Cálculo de Incerteza da Medição de Atenuação em Líquidos
}

\author{
Monique Kort-Kamp Figueiredo, MSc. \\ mkfigueiredo@inmetro.gov.br \\ Rodrigo Pereira Barreto da Costa-Félix, Dr. \\ rpfelix@inmetro.gov.br \\ André Victor Alvarenga, Dr. \\ avalvarenga@inmetro.gov.br \\ Luís Eduardo Maggi, MSc. \\ lemaggi@inmetro.gov.br \\ Gilberto Alves Romeiro, Dr. \\ gilbertoromeiro@ig.com.br
} Laboratório de Ultrassom, Diavi/Dimci/Inmetro, Duque de Caxias, RJ, Brasil, Instituto de Química, Universidade Federal Fluminense, Campos do Valonguinho, Niterói, RJ, Brasil,

\begin{abstract}
RESUMO
Incerteza é um parâmetro metrológico definido e inerente a qualquer experimento científico. Determinar, quantificar e combinar as incertezas melhora o padrão de qualidade e a confiabilidade metrológica dos resultados obtidos. Neste trabalho, a incerteza da medição de atenuação de líquidos foi estudada com o objetivo de se identificar os principais parâmetros que influenciam nesta incerteza. Para medir a atenuação utilizou-se o método ultrassônico de transmissão => recepção. Portanto, para cada frequência estudada, empregou-se um par de transdutores de mesma frequência nominal de ressonância. Foram utilizados 4 transdutores e 9 pontos de frequências variando entre $1.0 \mathrm{MHz}$ e $5.0 \mathrm{MHz}$. Para o arranjo experimental proposto, foram encontradas incertezas combinadas inferiores a $1 \%$ em todos os casos.
\end{abstract}

Palavras-chave: ultrassom, atenuação, incerteza de medição. 


\section{INTRODUÇÃO}

A incerteza de medição é um parâmetro, associado ao resultado de uma medição, seja ela uma calibração ou um ensaio, que caracteriza a dispersão dos valores que podem ser atribuídos ao mensurando (ABNT, INMETRO, 2003). A incerteza pode ser entendida como o grau de dúvida inerente ao processo de medição, no qual já não é mais possível a aplicação de correções para que a mesma seja eliminada por completo.

$\mathrm{Na}$ avaliação da conformidade, a incerteza vem sendo utilizada para definição de regras de decisão para aceitação e rejeição (ISO, 1998).

Vale lembrar que a incerteza é composta de diversos componentes, conforme sugere o GUM (ABNT, INMETRO, 2003), sendo, então, necessária uma avaliação completa e cautelosa de todas as suas fontes.

O ultrassom vem sendo aplicado em diversas etapas de processos químicos, quer seja na aceleração da reação (LIDA, TUZIUTI, YASUI, KOZUKA, TOWATA, 2008) quanto na separação de compostos ou mesmo na sua identificação e análise (GEORGOGIANNI, KONTOMINAS, TEGOU, AVLONITIS 2007). Outras atividades correlacionadas, tais como medição de vazão, também se mostram apropriadas à utilização do ultrassom como princípio físico de execução do processo. Entretanto, do ponto de vista metrológico, ainda há muito que se fazer.

Alguns trabalhos sobre a medição do coeficiente de atenuação e velocidade de propagação têm sido publicados, como os de Stavarache et al. (STAVARACHE, VINATORU, Y. MAEDA, 2007) e Bulent Koc (BULENT, 2009), porém estes apresentam apenas valores médios e desvio-padrão.

Assim, esse trabalho surge para corroborar e evidenciar cientificamente a utilização do ultrassom em sonoquímica e no controle de processos químicos de maneira mais abrangente.

\section{OBJETIVOS}

O objetivo deste trabalho é aplicar o método descrito no GUM para expressar as incertezas de medição da velocidade de propagação, viscosidade, amplitude do sinal entre outros parâmetros necessários para o cálculo do coeficiente de atenuação de líquidos, utilizando o ultrassom, através do método implementado no Laboratório de Ultrassom (Labus) do Inmetro.

\section{METODOLOGIA}

\subsection{Configuração experimental}

As amostras utilizadas para medição da atenuação foram inseridas em um cilindro de $20 \mathrm{~mm}$ de altura e $64 \mathrm{~mm}$ de diâmetro, sendo sua extremidade inferior vedada por uma película de PVC. A amostra de referência continha apenas água destilada e a amostra teste continha apenas glicerol a 99,5\%. Escolheu-se glicerol 99,5\% como amostra teste devido aos parâmetros dessa amostra ser de 
conhecimento prévio e fartamente difundido na literatura especializada (J.A. DEAN, Lange's Handbook of Chemistry). Foram utilizados pares de transdutores de frequência de ressonância nominal de $1,00 \mathrm{MHz}$ (frequência de $1,00 \mathrm{MHz}$ ), 2,25 MHz (frequências de 2,00 MHz, 2,25 MHz e 2,50 MHz), 3,50 MHz (frequências de $3,00 \mathrm{MHz}, 3,50 \mathrm{MHz}$ e $4,00 \mathrm{MHz}$ ) e $5,00 \mathrm{MHz}$ (frequências de $4,50 \mathrm{MHz}$ e $5,00 \mathrm{MHz}$ ). Todos os transdutores são da Panametrcis-NDT (OLYMPUS - NDT, Panametrics). Devido à baixa atenuação obtida utilizando os transdutores de $1,00 \mathrm{MHz}$, utilizou-se um cilindro de maiores dimensões $(50 \mathrm{~mm}$ de altura e $64 \mathrm{~mm}$ de diâmetro). Por outro lado, para os transdutores de $5,00 \mathrm{MHz}$ utilizou-se um cilindro de menor espessura (10 mm de altura e $64 \mathrm{~mm}$ de diâmetro), pois nesta freqüência a atenuação é muito grande, impossibilitando uma medição com suficiente acurácia em amostras com maior espessura, já que o sinal da amplitude torna-se muito pequeno.

As medições foram realizadas em duas etapas e o método utilizado foi o de transmissão $=>$ recepção. Na primeira etapa o cilindro contendo apenas água é posicionado entre dois transdutores, tal que um destes irá emitir o ultrassom e o outro irá receber o sinal transmitido através da amostra. Na segunda etapa o cilindro contendo a glicerina a $99,5 \%$ é posicionado entre os mesmos transdutores, na mesma distância.

Estas medições realizadas utilizando o cilindro com apenas água serão usadas como referência. llustra-se a montagem experimental na Figura 1.

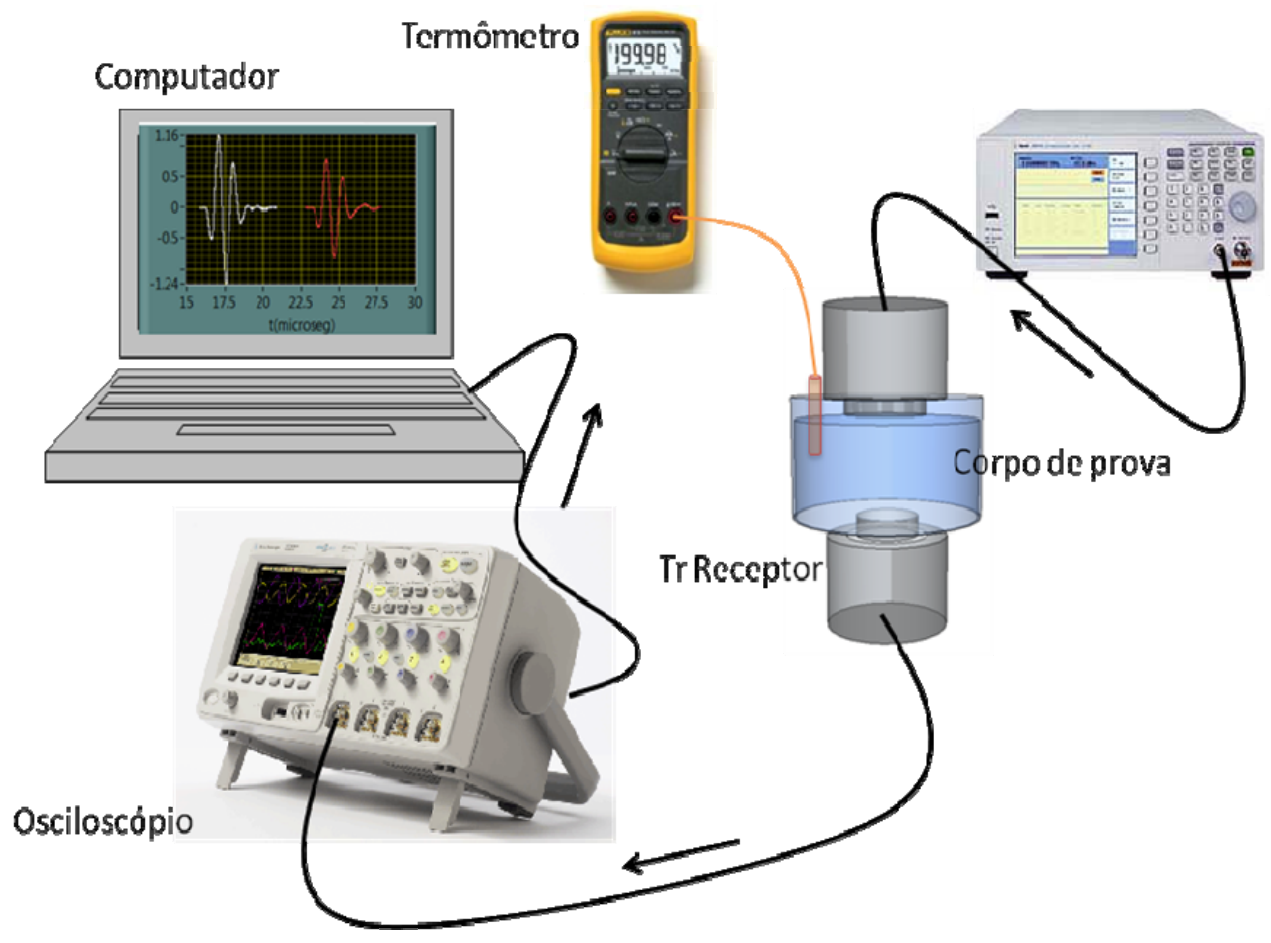

Figura 1: Montagem experimental utilizada na medição de atenuação, onde «Tr Emissor» é o transdutor emissor e «Tr Receptor» é o transdutor de recepção.

Todos os parâmetros utilizados no método implantado no Labus foram avaliados quanto suas incertezas padrão Tipo A e Tipo $B$, bem como a incerteza padrão combinada. O propósito de classificação Tipo A e Tipo B é o de indicar as duas maneiras diferentes de avaliar as componentes da incerteza. Na avaliação do Tipo A, o método de avaliação da incerteza baseia-se na análise estatística de uma 
série de observações. E na avaliação do Tipo $B$, o método de avaliação da incerteza baseia-se por outros meios que não a análise estatística de uma série de observações.

A incerteza padrão combinada de um resultado de medição é a incerteza padronizada quando este resultado é obtido por meio dos valores de várias outras grandezas, sendo igual à raiz quadrada positiva de uma soma de termos, sendo estes as variâncias ou covariâncias destas outras grandezas, ponderadas de acordo com quanto o resultado da medição varia com mudanças nestas grandezas.

\subsection{Formulação teórica}

\subsubsection{Formulação e cálculos de incerteza para encontrar a atenuação teórica}

A equação utilizada para o cálculo da atenuação teórica está descrita na equação (1).

$$
A T_{T}=\frac{20 \cdot \log \left\{\exp \left[x \alpha_{T}\right]\right\}}{x}
$$

onde $X$ é o comprimento da amostra e

$$
\alpha_{T}=\frac{(2 \pi \cdot f)^{2}}{2 \cdot \rho_{A} c_{A}^{3}}\left(\frac{4 \eta}{3}+\eta_{B}\right)
$$

Sendo $f$ a freqüência utilizada, $\eta$ é a viscosidade dinâmica, $\eta_{B}$ é a viscosidade de bulk, $\rho_{A}$ é a densidade da amostra e ${ }^{C_{A}}$ é a velocidade da amostra.

Para encontrar a incerteza da atenuação teórica de acordo com a equação (3), necessita-se do conhecimento das incertezas de todos os parâmetros utilizados na equação (1).

Nesta equação, as variáveis ${ }^{c}$ representam o coeficiente de sensibilidade de cada parâmetro e ${ }^{\mu}$ sua incerteza.

$$
\mu_{\alpha_{T}}^{2}=c_{f}^{2} \cdot \mu_{f}^{2}+c_{\eta}^{2} \cdot \mu_{\eta}^{2}+c_{\eta_{B}}^{2} \cdot \mu_{\eta_{B}}^{2}+c_{\rho_{A}}^{2} \cdot \mu_{\rho_{A}}^{2}+c_{c_{A}}^{2} \cdot \mu_{c_{A}}^{2}
$$

Para facilitar o cálculo dos coeficientes de sensibilidade realizou-se uma simplificação na fórmula teórica da atenuação (1), cujo resultado é apresentado na equação (4):

$$
A T_{T}=\frac{20(2 \pi \cdot f)^{2}}{2 \cdot \rho_{A} c_{A}^{3}}\left(\frac{4 \eta}{3}+\eta_{B}\right) \cdot \log e
$$


sendo

$$
\begin{aligned}
& \eta_{B}=1,03 . \eta \therefore \eta_{E}=\frac{4}{3} \eta+\eta_{B}=\frac{4}{3} \eta+1,03 . \eta \\
& \therefore \eta_{E}=\frac{7,09 \eta}{3}
\end{aligned}
$$

E assim, pode-se reescrever a equação (4) como:

$$
A T_{T}=\frac{20.4 \pi^{2} \cdot 7,09 \cdot \log e}{2 \cdot 3} \cdot \frac{f^{2} \cdot \eta_{E}}{\rho_{A} \cdot c_{A}^{3}}
$$

Pode-se, assim, ter um coeficiente de sensibilidade e uma incerteza para os valores numéricos (constantes), como demonstrado na equação (7), simplificando a fórmula teórica.

$$
c_{0}=\frac{20.4 \pi^{2} \cdot 7,09 \cdot \log e}{2 \cdot 3} ; \mu_{0}=0
$$

A fórmula simplificada então está apresentada na equação (8).

$$
A T_{T}=c_{0} \cdot \frac{f^{2} \cdot \eta_{E}}{\rho_{A} \cdot c_{A}^{2}}
$$

Sendo assim, a fórmula utilizada para encontrar a incerteza da atenuação teórica será:

$$
\mu_{\alpha_{T}}^{2}=c_{f}^{2} \cdot \mu_{f}^{2}+c_{\eta_{E}}^{2} \cdot \mu_{\eta_{E}}^{2}+c_{\rho_{A}}^{2} \cdot \mu_{\rho_{A}}^{2}+c_{c_{A}}^{2} \cdot \mu_{c_{A}}^{2}
$$

\section{Coeficientes de sensibilidade utilizados nos cálculos da atenuação} teórica

Os coeficientes de sensibilidade são obtidos derivando-se parcialmente a equação (8), conforme apresentado nas equações (10-13).

$$
\begin{gathered}
{ }_{c_{f}}=\frac{\delta A T_{T}}{\delta f}=\left(\frac{2 \cdot c_{0} \cdot f \cdot \eta}{\rho_{A} \cdot c_{A}^{3}}\right), \\
{ }^{c}=\frac{\delta A T_{T}}{\delta \eta}=\left(\frac{c_{0} \cdot f^{2}}{\rho_{A} \cdot c_{A}^{3}}\right) \\
{ }_{\rho_{A}}=\frac{\delta A T_{T}}{\delta \rho_{A}}=\left(\frac{-c_{0} \cdot f^{2} \cdot \eta}{\rho_{A}^{2} \cdot c_{A}^{3}}\right)
\end{gathered}
$$




$$
{ }^{c} C_{A}=\frac{\delta A T_{T}}{\delta c_{A}}=\left(\frac{-3 \cdot c_{0} \cdot f^{2} \cdot \eta}{\rho_{A} \cdot c_{A}^{4}}\right)
$$

Incertezas padrão do Tipo A e B e a incerteza padrão combinada

Para a frequência utilizou-se apenas a incerteza padrão do tipo B:

$$
\mu_{f}=\left(\frac{\text { resol. }}{2 \sqrt{3}}\right)
$$

onde «resol.» é a resolução do instrumento utilizado para gerar o sinal, neste caso, o gerador de sinais modelo 33250A (Agilent Tecnologies, CA, EUA). O fator de $2 \sqrt{3}$ é utilizado, levando-se em consideração que as medições realizadas por este equipamento fornecem resultados que seguem uma distribuição retangular.

Para as variáveis $\rho_{A}$ e $\eta$ foram utilizadas as incertezas padrão do Tipo A, obtidas por meio do cálculo do desvio-padrão de cinco medições. O valor de ${ }^{c_{A}}$ é obtido pela equação (15).

$$
v=\frac{x}{t}
$$

Logo, para se estimar a incerteza da velocidade da amostra, precisa-se conhecer as incertezas da espessura da amostra $\left({ }^{x}\right)$ e o tempo $\left({ }^{t}\right)$ que a onda demorou para percorrer uma determinada espessura. Sendo assim, a incerteza da velocidade é dada pela equação (16).

$$
\mu_{v}^{2}=c_{x}^{2} \cdot \mu_{x}^{2}+c_{t}^{2} \cdot \mu_{t}^{2}
$$

Coeficientes de sensibilidade utilizados nos cálculos da velocidade

Os coeficientes de sensibilidade são obtidos derivando-se parcialmente a equação (15), conforme apresentado nas equações (17-18).

$$
\begin{gathered}
c_{x}=\left(\frac{\partial v}{\partial x}\right)=\left(\frac{1}{t}\right) \\
c_{t}=\left(\frac{\partial v}{\partial t}\right)=\left(\frac{-x}{t^{2}}\right)
\end{gathered}
$$

Incertezas-padrão do tipo A e B, bem como a incerteza combinada para o cálculo da incerteza da velocidade

Para as variáveis ${ }^{x}$ e $t$, foram utilizadas a incerteza padrão do Tipo $A$, obtida por meio do cálculo do desvio-padrão de cinco medições. As incertezas do Tipo B de $t$ foram baseadas na informação do osciloscópio modelo DSO6032A (AGILENT TECHNOLOGIES, CA, USA), utilizado no experimento, conforme apresentado nas equações (19-20). 


$$
\begin{gathered}
\mu_{t_{B 1}}=2 \cdot\left(\frac{\mu \Delta t}{2 \sqrt{3}}\right) \\
\mu_{t_{B 2}}=( \pm\{0,0015 \% \text {. leitura do tempo } \pm 0,1 \% 5 \mu \mathrm{s} \pm 20 \mathrm{ps}\}) \\
\mu_{\text {comb }}^{2}=\mu_{t_{A}}^{2}+\mu_{t_{B 1}}^{2}+\mu_{t_{B 2}}^{2}
\end{gathered}
$$

3.3.2 Formulação e cálculos de incerteza para encontrar a atenuação experimental.

A equação utilizada para o cálculo da atenuação experimental está descrita na equação (22). Onde V0 é a amplitude do sinal de referencia (no caso água), V é a amplitude do sinal da amostra, e $x_{e}$ é a espessura da amostra, no caso glicerina 99,5\%.

$$
A T_{E}=\frac{20 \log \left(\frac{V_{0}}{V}\right)}{x_{e}}
$$

Para encontrar a incerteza da atenuação experimental de acordo com a equação (23), necessita-se do conhecimento das incertezas de todos os parâmetros utilizados na equação (22).

$$
\mu_{A T_{E}}^{2}=c_{x_{e}}^{2} \cdot \mu_{x_{e}}^{2}+c_{V_{0}}^{2} \cdot \mu_{V_{0}}^{2}+c_{V}^{2} \cdot \mu_{V}^{2}
$$

Coeficientes de sensibilidade dos parâmetros utilizados nos cálculos da atenuação experimental.

Os coeficientes de sensibilidade são obtidos derivando-se parcialmente a equação (22), conforme apresentado nas equações (24-26).

$$
\begin{aligned}
& c_{X_{e}}=\frac{\delta A T_{E}}{\delta x_{e}}=\frac{-20 \ln \left(V_{0} / V\right)}{\ln (10) \cdot x_{e}^{2}} \\
& c_{V}=\frac{\delta A T_{E}}{\delta V}=\frac{-20}{x_{e} \cdot V \cdot \ln (10)} \\
& c_{V_{0}}=\frac{\delta A T_{E}}{\delta V_{0}}=\frac{20}{x_{e} \cdot V_{0} \cdot \ln (10)}
\end{aligned}
$$

Coeficiente de sensibilidade dos parâmetros utilizados nos cálculos da atenuação experimental.

Para a variável ${ }^{x_{e}}$ utilizou-se a mesma incerteza calculada para a espessura ${ }^{x}$ ) no cálculo da atenuação teórica. Para as variáveis $V \mathrm{e}^{V_{0}}$, utilizou-se a incerteza padrão do Tipo A, obtida por meio do cálculo do desvio-padrão de cinco medições. As incertezas do Tipo B são demonstradas a seguir: 


$$
\begin{gathered}
\mu_{V_{B_{1}}}=\left(\left(\frac{V_{\max .}}{2^{8}}\right) \cdot \frac{1}{2 \sqrt{3}}\right) \\
\left.\mu_{V_{B 2}}=( \pm\{2,2 \% \text {. escala completa(8.V/Div. })\}\right) \\
\mu_{\text {com. }}^{2}=\mu_{V_{A}}^{2}+\mu_{V_{B 1}}^{2}+\mu_{V_{B 2}}^{2}
\end{gathered}
$$

\section{APRESENTAC̣̃̃O DOS DADOS E DISCUSSÃO DOS RESULTADOS}

Utilizando-se os parâmetros tais como definidos nas equações (1) e (22), os resultados das medições de atenuação teórica e experimental, realizadas com a amostra padrão de glicerina 99,5\%, se encontram na Tabela 1 expressa em dB/cm. Nela pode-se observar que os resultados teóricos e experimentais são equivalentes para todas as frequências nominais de ressonância utilizadas. A viscosidade é dependente diretamente da temperatura e, portanto foi corrigida em todas as medições variando entre $19^{\circ} \mathrm{C}$ e $23^{\circ} \mathrm{C}$. As incertezas padrão combinadas das variáveis que descrevem os mensurandos em questão, tanto para os cálculos da atenuação teórica quanto experimental, estão descritas na Tabela 2.

Tabela 1- Resultado da média das medições para os cálculos da atenuação teórica e experimental.

\begin{tabular}{|c|c|c|c|c|c|c|c|c|}
\hline \multirow{2}{*}{$\begin{array}{c}f \\
{[\mathbf{M H z}]}\end{array}$} & \multirow{2}{*}{\begin{tabular}{c}
\multicolumn{3}{|c|}{ TEÓRICO } \\
{$[\mathbf{c m}]$}
\end{tabular}} & \multicolumn{3}{|c|}{} & \multicolumn{3}{c|}{ EXPERIMENTAL } \\
\cline { 3 - 9 } & & $\begin{array}{c}C_{A} \\
{[\mathbf{m} / \mathbf{s}]}\end{array}$ & $\begin{array}{c}\eta_{E} \\
{[\mathbf{m . P a}]}\end{array}$ & $\begin{array}{c}\alpha_{T} \\
{\left[\mathbf{m}^{-1}\right]}\end{array}$ & $\begin{array}{c}A T \\
{[\mathbf{d B} / \mathbf{c m}]}\end{array}$ & $\begin{array}{c}V_{0} \\
{[\mathbf{V}]}\end{array}$ & $\begin{array}{c}V \\
{[\mathbf{V}]}\end{array}$ & $\begin{array}{c}A T_{E} \\
{[\mathbf{d B} / \mathbf{c m}]}\end{array}$ \\
\hline $\mathbf{1 . 0 0}$ & $\mathbf{5 . 6 2}$ & 1885.2 & 1.0744 & 5.9 & 0.51 & 3.247 & 2.266 & 0.55 \\
\hline $\mathbf{2 . 0 0}$ & $\mathbf{1 . 9 9}$ & 1888.4 & 1.1401 & 24.7 & 2.15 & 4.603 & 2.709 & 2.3 \\
\hline $\mathbf{2 . 2 5}$ & $\mathbf{2 . 0 0}$ & 1880.1 & 1.1669 & 32.4 & 2.82 & 4.906 & 2.569 & 2.81 \\
\hline $\mathbf{2 . 5 0}$ & $\mathbf{1 . 9 8}$ & 1885.4 & 1.1042 & 37.6 & 3.27 & 4.28 & 2.144 & 3.07 \\
\hline $\mathbf{3 . 0 0}$ & $\mathbf{2 . 1 4}$ & 1895.6 & 1.087 & 52.4 & 4.56 & 0.808 & 0.271 & 4.42 \\
\hline $\mathbf{3 . 5 0}$ & $\mathbf{2 . 1 2}$ & 1896.1 & 1.1512 & 75.5 & 6.56 & 1.13 & 0.244 & 6.24 \\
\hline $\mathbf{4 . 0 0}$ & $\mathbf{1 . 7 2}$ & 1881 & 0.9954 & 86.8 & 7.54 & 1.053 & 0.309 & 6.96 \\
\hline $\mathbf{4 . 5 0}$ & $\mathbf{1 . 5 9}$ & 1882.9 & 0.9825 & 109.3 & 9.44 & 0.783 & 0.171 & 8.82 \\
\hline $\mathbf{5 . 0 0}$ & $\mathbf{1 . 1 9}$ & 1913.3 & 0.994 & 122.05 & 10.4 & 0.517 & 0.198 & 9.42 \\
\hline
\end{tabular}

As incertezas-padrão combinadas tanto da medição da atenuação teórica quanto da experimental obtiveram consideráveis resultados para todas as faixas de frequências testadas, como mostra a Tabela 2. Comparando-se os resultados apresentados nestas tabelas, pode-se afirmar que a incerteza do cálculo da atenuação experimental é maior do que a incerteza do cálculo da atenuação teórica, como o esperado.

Conforme sugerido nos manuais metrológicos, uma avaliação detalhada da incerteza de todos os parâmetros envolvidos na medição possibilita uma análise mais detalhada dos resultados, permitindo identificar com maior exatidão onde pode 
estar localizado um possível problema e corrigi-lo. Desta forma, os experimentos podem ser aprimorados fornecendo assim resultados mais confiáveis.

Analisando-se a Tabela 2, verifica-se que a incerteza-padrão combinada da velocidade é a incerteza que mais contribui para a incerteza-padrão combinada do cálculo da atenuação teórica. O valor dessa grandeza foi obtido utilizando a equação (16). Nesta, a incerteza-padrão do Tipo A do tempo de propagação para a glicerina 99,5\% representa a maior contribuição para a incerteza final. Esse fato pode ser atribuído, em parte, ao procedimento de substituição da amostra de referência pela amostra de glicerina, o que pode causar um pequeno desalinhamento entre os transdutores. Melhorando-se a reprodutibilidade desse procedimento seria possível diminuir a incerteza da velocidade. Outro fator importante que pode ser observado nessas tabelas é contribuição da viscosidade na incerteza do cálculo da atenuação teórica, sendo este o segundo fator mais importante. O valor dessa grandeza foi obtido através da temperatura, uma vez que a viscosidade é dependente da temperatura. A utilização de termômetros calibrados e a realização das medições em temperaturas controladas podem resultar em diminuição da incerteza da viscosidade.

Para o cálculo de atenuação experimental, observa-se nas tabelas que a incerteza-padrão combinada da amplitude do sinal $(V)$ é a variável que mais contribui para a incerteza do cálculo da atenuação experimental. $E$ isso ocorre devido à atenuação na glicerina ser grande e quando se obtém uma amplitude do sinal em [mV], aumenta-se consideravelmente a incerteza do sinal e consequentemente aumenta também a incerteza do cálculo da atenuação experimental. Pode-se observar, também, que com o aumento da frequência contribui para o aumento da incerteza de medição da atenuação. Porém, existem alguns casos que não seguem essa regra, devido à amplitude do sinal recebido após propagação através da amostra ser de menor intensidade, mesmo utilizandose o cilindro com água. 
Tabela 2 - Componentes da incerteza padrão combinada dos cálculos da atenuação teórica e experimental da glicerina 99,5\% nas frequências de 1 a $5 \mathrm{MHz}$.

\begin{tabular}{|c|c|c|c|c|c|c|c|c|c|c|}
\hline \multirow{2}{*}{$\begin{array}{c}\text { Frequência } \\
\text { (MHz) }\end{array}$} & \multirow{2}{*}{ Componente } & \multicolumn{5}{|c|}{ TEÓRICO } & \multicolumn{4}{|c|}{ EXPERIMENTAL } \\
\hline & & & $\eta_{E}$ & $\rho_{A}$ & $C_{A}$ & $A T_{T}$ & $V_{0}$ & $V$ & $X$ & $A T_{E}$ \\
\hline \multirow{3}{*}{1.00} & $\mu_{i}$ & $8.3 \times 10^{-14}$ & $5.7 \times 10^{-5}$ & $1.6 \times 10^{-10}$ & $2.3 \times 10^{4}$ & & $4.1 \times 10^{-2}$ & $3.8 \times 10^{-2}$ & $4.2 \times 10^{-6}$ & \\
\hline & $C_{i}$ & $1.1 \times 10^{-12}$ & $2.3 \times 10^{-3}$ & $1.6 \times 10^{-1}$ & $6.6 \times 10^{-11}$ & & $2.3 \times 10^{-1}$ & $4.6 \times 10^{-1}$ & $9.8 \times 10^{-3}$ & \\
\hline & $\left(\mu_{i}\right)\left(c_{i}\right)$ & $8.7 \times 10^{-26}$ & $1.3 \times 10^{-7}$ & $2.6 \times 10^{-11}$ & $1.5 \times 10^{-06}$ & $1.3 \times 10^{-3}$ & $9.2 \times 10^{-3}$ & $1.8 \times 10^{-2}$ & $4.1 \times 10^{-8}$ & $1.6 \times 10^{-01}$ \\
\hline \multirow{3}{*}{2.00} & $\mu_{i}$ & $8.3 \times 10^{-14}$ & $5.7 \times 10^{-5}$ & $1.6 \times 10^{-10}$ & $1.4 \times 10^{3}$ & & $1.1 \times 10^{-01}$ & $3.7 \times 10^{-2}$ & $3.8 \times 10^{-09}$ & \\
\hline & $C_{i}$ & $4.7 \times 10^{-12}$ & $3.6 \times 10^{-2}$ & 2.98 & $1.2 \times 10^{-9}$ & & 0.89 & 2.57 & $3.3 \times 10^{2}$ & \\
\hline & $\left(\mu_{i}\right)\left(c_{i}\right)$ & $3.9 \times 10^{-25}$ & $2.1 \times 10^{-06}$ & $4.7 \times 10^{-10}$ & $1.7 \times 10^{-06}$ & $1.9 \times 10^{-03}$ & $9.6 \times 10^{-02}$ & $9.4 \times 10^{-02}$ & $1.2 \times 10^{-08}$ & $4.3 \times 10^{-01}$ \\
\hline \multirow{3}{*}{2.25} & $\mu_{i}$ & $8.3 \times 10^{-14}$ & $5.7 \times 10^{-5}$ & $1.6 \times 10^{-10}$ & $2.1 \times 10^{5}$ & & $1.1 \times 10^{-01}$ & $3.8 \times 10^{-2}$ & $1.2 \times 10^{-10}$ & \\
\hline & $C_{i}$ & $6.4 \times 10^{-12}$ & $5.9 \times 10^{-2}$ & 5.13 & $2.1 \times 10^{-9}$ & & $7.8 \times 10^{-1}$ & 2.85 & $5.1 \times 10^{3}$ & \\
\hline & $\left(\mu_{i}\right)\left(c_{i}\right)$ & $5.3 \times 10^{-25}$ & $3.4 \times 10^{-06}$ & $8.1 \times 10^{-10}$ & $4.3 \times 10^{-04}$ & $2.1 \times 10^{-02}$ & $9.3 \times 10^{-02}$ & $1.1 \times 10^{-01}$ & $6.0 \times 10^{-08}$ & $4.5 \times 10^{-01}$ \\
\hline \multirow{3}{*}{2.50} & $\mu_{i}$ & $8.3 \times 10^{-14}$ & $5.7 \times 10^{-5}$ & $1.6 \times 10^{-10}$ & $1.6 \times 10^{5}$ & & $1.2 \times 10^{-01}$ & $3.2 \times 10^{-2}$ & $2.0 \times 10^{-10}$ & \\
\hline & $C_{i}$ & $7.1 \times 10^{-12}$ & $9.0 \times 10^{-2}$ & 6.97 & $2.8 \times 10^{-9}$ & & 1.04 & 4.05 & $5.4 \times 10^{3}$ & \\
\hline & $\left(\mu_{i}\right)\left(c_{i}\right)$ & $5.9 \times 10^{-25}$ & $5.2 \times 10^{-06}$ & $1.1 \times 10^{-9}$ & $4.5 \times 10^{-04}$ & $2.2 \times 10^{-02}$ & $1.2 \times 10^{-01}$ & $1.3 \times 10^{-01}$ & $1.1 \times 10^{-07}$ & $5.0 \times 10^{-01}$ \\
\hline \multirow{3}{*}{3.00} & $\mu_{i}$ & $8.3 \times 10^{-14}$ & $5.7 \times 10^{-5}$ & $1.6 \times 10^{-10}$ & $2.2 \times 10^{5}$ & & $6.9 \times 10^{-03}$ & $3.7 \times 10^{-4}$ & $7.8 \times 10^{-11}$ & \\
\hline & $C_{i}$ & $9.4 \times 10^{-12}$ & $1.8 \times 10^{-1}$ & 13.4 & $5.3 \times 10^{-9}$ & & 25 & $2.2 \times 10^{3}$ & $1.2 \times 10^{-1}$ & \\
\hline & $\left(\mu_{i}\right)\left(c_{i}\right)$ & $7.9 \times 10^{-25}$ & $1.0 \times 10^{-05}$ & $2.1 \times 10^{-9}$ & $1.2 \times 10^{-03}$ & $3.5 \times 10^{-02}$ & $1.7 \times 10^{-01}$ & $8.3 \times 10^{-02}$ & $9.3 \times 10^{-12}$ & $5.1 \times 10^{-01}$ \\
\hline \multirow{3}{*}{3.50} & $\mu_{i}$ & $8.3 \times 10^{-14}$ & $5.7 \times 10^{-5}$ & $1.6 \times 10^{-10}$ & $1.8 \times 10^{5}$ & & $7.4 \times 10^{-03}$ & $3.7 \times 10^{-4}$ & $2.8 \times 10^{-11}$ & \\
\hline & $c_{i}$ & $1.4 \times 10^{-11}$ & $3.3 \times 10^{-1}$ & 27.8 & $1.1 \times 10^{-8}$ & & 13 & $2.6 \times 10^{2}$ & $3.6 \times 10^{3}$ & \\
\hline & $\left(\mu_{i}\right)\left(c_{i}\right)$ & $1.2 \times 10^{-24}$ & $1.9 \times 10^{-05}$ & $4.4 \times 10^{-9}$ & $2.0 \times 10^{-03}$ & $4.5 \times 10^{-02}$ & $9.6 \times 10^{-02}$ & $9.7 \times 10^{-02}$ & $1.0 \times 10^{-5}$ & $4.4 \times 10^{-01}$ \\
\hline \multirow{3}{*}{4.00} & $\mu_{i}$ & $8.3 \times 10^{-14}$ & $5.7 \times 10^{-5}$ & $1.6 \times 10^{-10}$ & $3.7 \times 10^{5}$ & & $7.9 \times 10^{-03}$ & $3.1 \times 10^{-4}$ & $1.1 \times 10^{-8}$ & \\
\hline & $C_{i}$ & $1.4 \times 10^{-11}$ & $5.8 \times 10^{-1}$ & 36.7 & $1.5 \times 10^{-8}$ & & 29.2 & $3.4 \times 10^{2}$ & $6.1 \times 10^{2}$ & \\
\hline & $\left(\mu_{i}\right)\left(c_{i}\right)$ & $1.2 \times 10^{-24}$ & $3.4 \times 10^{-05}$ & $5.8 \times 10^{-9}$ & $5.5 \times 10^{-03}$ & $7.5 \times 10^{-02}$ & $2.3 \times 10^{-01}$ & $1.0 \times 10^{-01}$ & $7.0 \times 10^{-6}$ & $5.8 \times 10^{-01}$ \\
\hline \multirow{3}{*}{4.50} & $\mu_{i}$ & $8.3 \times 10^{-14}$ & $5.7 \times 10^{-5}$ & $1.6 \times 10^{-10}$ & $4.8 \times 10^{5}$ & & $5.0 \times 10^{-04}$ & $3.3 \times 10^{-4}$ & $1.3 \times 10^{-9}$ & \\
\hline & $C_{i}$ & $1.8 \times 10^{-11}$ & $9.4 \times 10^{-1}$ & 57.5 & $2.3 \times 10^{-8}$ & & $5.5 \times 10^{2}$ & $1.1 \times 10^{3}$ & $8.6 \times 10^{2}$ & \\
\hline & $\left(\mu_{i}\right)\left(c_{i}\right)$ & $1.5 \times 10^{-24}$ & $5.7 \times 10^{-05}$ & $9.1 \times 10^{-9}$ & $1.1 \times 10^{-02}$ & $1.1 \times 10^{-01}$ & $2.8 \times 10^{-02}$ & $3.8 \times 10^{-01}$ & $1.1 \times 10^{-6}$ & $6.4 \times 10^{-01}$ \\
\hline \multirow{3}{*}{5.00} & $\overline{\mu_{i}}$ & $8.3 \times 10^{-14}$ & $5.7 \times 10^{-5}$ & $1.6 \times 10^{-10}$ & $7.3 \times 10^{9}$ & & $4.5 \times 10^{-04}$ & $3.4 \times 10^{-4}$ & $7.5 \times 10^{-10}$ & \\
\hline & $c_{i}$ & $1.8 \times 10^{-11}$ & 1.16 & 72.4 & $1.1 \times 10^{-11}$ & & $2.8 \times 10^{2}$ & $1.9 \times 10^{3}$ & $6.7 \times 10^{1}$ & \\
\hline & $\left(\mu_{i}\right)\left(c_{i}\right)$ & $1.5 \times 10^{-24}$ & $6.7 \times 10^{-05}$ & $1.1 \times 10^{-8}$ & $7.8 \times 10^{-02}$ & $2.8 \times 10^{-01}$ & $1.3 \times 10^{-01}$ & $6.7 \times 10^{1}$ & $5.0 \times 10^{-8}$ & $8.9 \times 10^{-01}$ \\
\hline
\end{tabular}


A Figura 2 mostra a relação entre a frequência e a atenuação, e suas respectivas incertezas, onde se pode observar que a atenuação aumenta em função da frequência e, de forma geral, a incerteza também segue essa tendência. Todavia, os valores de incerteza encontrados no cálculo experimental, estão associados às diferenças na amplitude do sinal, que pode variar dependendo da frequência nominal de ressonância de cada transdutor.

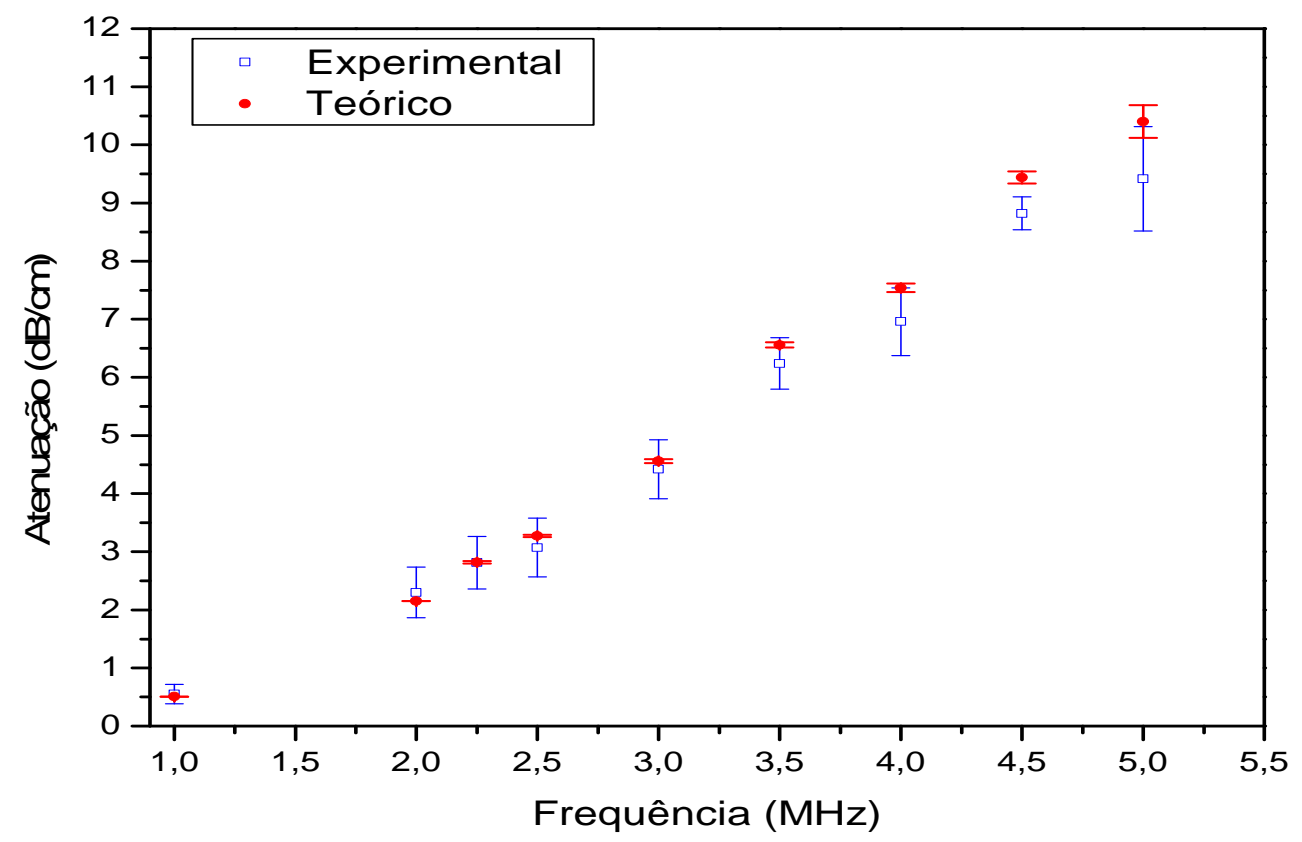

Figura 2 - Gráfico do coeficiente de atenuação experimental e teórico em função da frequência com suas respectivas incertezas.

\section{CONCLUSÃO}

Foi realizado um estudo da incerteza na medição de atenuação em líquidos (glycerol), a partir de cálculos teóricos e experimentais. Constatou-se que, para a atenuação teórica, a maior fonte de incerteza é devido à medição da velocidade e da viscosidade. No caso da velocidade, a maior contribuição para a incerteza foi devido à variabilidade na captação do tempo de pico.

Para a atenuação experimental, a maior incerteza é devido à medição da amplitude do sinal, uma vez que quanto maior for à atenuação na amostra menor será o sinal e, conseqüentemente, maior será a incerteza.

\section{ABSTRACT}

Uncertainty is a metrological parameter defined and intrinsic in any scientific experiment. Determine, quantify and combine uncertainties improves the standard of quality and metrological reliability of the results. In this work, the uncertainty of measurement of attenuation of fluid was studied with aiming of identify the main parameters that influence this uncertainty. To measure the attenuation, it was used the ultrasonic transmission=> reception method. Therefore, for each frequency 
studied, it was used a pair of transducers of the same nominal frequency of resonance. It was used four transducers and nine points of frequencies ranging between $1.0 \mathrm{MHz}$ and $5.0 \mathrm{MHz}$ for the experimental arrangement proposed, it was found combined uncertainty less than $1 \%$ in all cases.

Keywords: ultrasound, attenuation, measurement uncertainty.

\section{REFERÊNCIAS}

ABNT /INMETRO. Guia para a Expressão da Incerteza de Medição. Terceira edição brasileira em língua portuguesa. Rio de Janeiro: ABNT, INMETRO, 120 p. 2003.

AGILENT, EUA, Gerador de sinais modelo 33250A. Manual do usuário.

AGILENT TECHNOLOGIES, Osciloscópio de armazenamento digital Série DSO6032A. Manual do usuário.

BULENT, A.K.; "Ultrasonic monitoring of glycerol settling during transesterification of soybean oil", Bioresource Technology, vol. 100, pp. 19-24, 2009.

DASH, J.K.; SAHU, M.; CHA.KRABORTTY. M.; (2000)"Viscosity, conductance and acoustic studies on lanthanum (hi) chloride in water and maltose-water mixed solvent system" Journal of Molecular Liquids , 84, 215-222, 2000.

GEORGOGIANNI, K. G.; KONTOMINAS, M. G.; TEGOU, E.; AVLONITIS "Biodiesel production: reaction and process parameters of alkali-catalyzed transesterification of waste frying oils" Energy \& Fuels, 21, 3023-3027, 2007.

ISO - Geometrical Product Specification (GPS) - Inspection by measurement of workpieces and measuring equipment; Part 1: Decision rules for proving conformance or nonconformance with specifications, 14253 - 1. Geneva 1998.

J.A. DEAN, Lange's Handbook of Chemistry, 13th Edition, McGraw-Hill Book, New York, pp 10-97.

LIDA, Y., TUZIUTI, T., YASUI, K., KOZUKA, T., TOWATA, A. "Protein release from yeast cells as an evaluation method of physical effects in ultrasonic field" Ultrasonics Sonochemistry, v. 15, p. 995-1000.; 2008.

OLYMPUS - NDT, Panametrics - NDT Ultrasonic Transducers for Nondestructive Testing, Immersion Transducer VIDEOSCAN V306-SU.

STAVARACHE, C.,VINATORU, M., MAEDA, Y. "Aspects of ultrasonically assisted transesterification of various vegetableoils with methanol", Ultrasonics Sonochemistry, vol. 14, pp. 380-386, 2007. 